\title{
Data Aggregation Scheme for a Multi-Application WSN
}

\author{
Ahmad Sardouk, Rana Rahim-Amoud, Leïla Merghem-Boulahia, \\ and Dominique Gaïti \\ ICD/ERA, FRE CNRS 2848, Université de Technologies de Troyes, \\ 12 rue Marie Curie, 10010 Troyes Cedex, France \\ \{ahmad.sardouk, rana.amoud, leila.boulahia, dominique.gaiti\}@utt.fr
}

\begin{abstract}
Wireless Sensor Networks (WSNs) are still designed and deployed for one specific application, while it is vital to deploy several applications over the same WSN in order to reduce the deployment and the administrative costs. One of the remaining problems, in this domain, resides in the data aggregation solutions, which are proposed generally for one application and may drain the WSN power in a multi-application context. Therefore, we propose a data aggregation scheme based on a multi-agent system to aggregate the WSN information in an energyefficient manner even if we are deploying several applications over this network. This proposal has proved its performance in the context of one and several applications through successive simulations in different network scales.
\end{abstract}

\section{Introduction}

The basic role of a Wireless Sensor Network is to collect information from the environment by many sensor nodes (SNs). Even due to hardware limits the WSNs have been predominantly tasked with a single application in the past [1]. The recent advances, in the hardware part of an SN, have lead to the creation of more powerful SNs. For instance, the SNs produced by Sun (Sun SPOT) [2] are a good example of these advances, where each SN has a $180 \mathrm{Mhz}, 512 \mathrm{~KB}$ of RAM and $4 \mathrm{MB}$ of flash memory. These advances have allowed/pushed a growing interest in deploying several applications over the same WSN.

The solutions proposed to deploy several applications could be divided into two categories. The first one [3] [4] proposes to change the code of the running application if needed. The second category [1] 5] manages the access of the different applications to the SN resources (CPU and Memory).

The SNs have a finite battery life and nodes failures can lead to a loss of data or network partition. Therefore, in addition to these kinds of solutions, we need to optimize the power consumption of the $\mathrm{SNs}$, which is mainly related to the information communication.

In this paper, we propose an energy-efficient data gathering scheme, which optimizes the power consumption of WSNs deployed for one or several applications. This solution is based on a multi-agent system (MAS) to reduce the

T. Pfeifer and P. Bellavista (Eds.): MMNS 2009, LNCS 5842, pp. 18:-188, 2009.

(C) IFIP International Federation for Information Processing 2009 
amount of the gathered information by treating and filtering the information locally at the SNs. Moreover, The deployed MAS will be empowered by a strategy allowing the agent to make more appropriate decisions and to prioritize a running application in favor of another one.

The remaining of this paper is organized as follows. In section 2 , we present our proposal and how the agent makes its cooperation decisions. Next, section 3 explains our simulations results. Finally, the conclusion is given in section 4 .

\section{Multi-Application WSN Data Aggregation (MADA)}

\subsection{System Design}

In our proposal we implement an agent in each SN. Each agent will be able to control the power and radio entities of its SN. The proposed architecture is a two layers architecture and it is illustrated in Fig. 1. The low layer is constituted by the physical SNs and the high layer is formed by the agents.

Each agent manages several types of applications. For example, if we want to deploy two applications (monitoring and tracking), each agent will create two executive agents (EA): monitoring agent (MA) and tracking agent (TA). Once the work of an EA is acheived, it will be killed by the main agent, which created it. Hence, the role of the main agent will be to create EAs, attach them to applications, and then to kill them at the end of their tasks.

\subsection{Agent States}

Fig. 2 presents the state diagram of a typical EA in our proposal. The events are the transitions that allow an EA to pass from one state to another one.

To illustrate our proposal, we will use Fig. 1 as a reference and we will suppose that we have two simultaneously running applications (1) Monitoring application which corresponds to a fire detected by the node A (2) Tracking application related to a desired object detected by node $\mathrm{X}$. We mention also that $\mathrm{A}, \mathrm{B}$ and $\mathrm{X}$ are neighbor nodes. In the "listening" state (partially managed by the main agent), the agent waits for an interruption that could come from one of two main sources. The first one is when the sensing entity perceives new information from the environment (e.g., sensors of node A detect a fire or sensors of node X detect an object). This information will be sent to the corresponding agent, which will create an MA if it is not previously created. This MA passes to a "processing" state to treat the received information and to judge its importance degree. The second type of interruption is a cooperation request message sent by a neighbor agent (or EA). For example, receiving a cooperation request, from a $T A_{x}$, asking if this node has detected a defined object. Thus, the corresponding agent creates a TA for this tracking application. Then, the created TA passes to a "joining a cooperation" state to decide to participate in this cooperation or not.

In the "processing" state, the EA treats the perceived information and following its importance degree, a cooperation could be initiated. For example, for the 


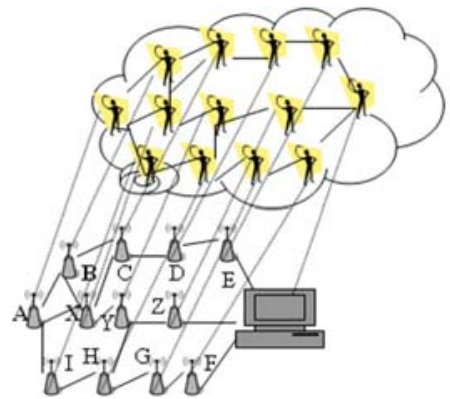

Fig. 1. Two Layers Architecture

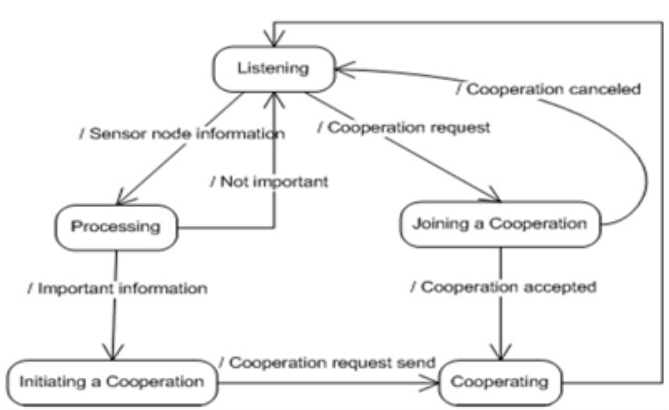

Fig. 2. EA state diagram

monitoring application, the MA of A compares the detected temperature value with the previous one and finds a big difference. The MA considers that there is an weird event (like a fire). Based on the processing result, the EA can judge the information importance degree, which will be identified later.

The "processing" triggers "initiating a cooperation" state if it estimates the perceived information as important. Thus, the EA cooperates with other EAs in order to execute the task related to this information. For example, the TA of X sends the detected information to its one-hop neighbors to inform them about the existance of the desired object in its area and at which distance and when it was detected. The goal of sharing its information is to localize the object, which needs at least three EAs. This state appears as an intermediate state, it allows an EA to start cooperation and then it passes to a "cooperating state" to manage this cooperation.

"Joining a cooperation" state starts when an EA receives a cooperation request from another EA. For example, the MAs of agents B and X, which are neighbors of agent $\mathrm{A}$, have received a cooperation request from the MA of $\mathrm{A}$ asking about their perceived temperatures. If the MAs of B and X have detected an important temperature variation, they send their information to A. Simultaneously, the TA of A has received a cooperation request from the TA of X concerning the object detection. If the TA of A has received similar signal, it sends the information obtained from the signal (estimated distance and detection time) to the TA of X. The "cooperating" state allows the EAs to communicate together. It is trigged from the "initiating a cooperation" state if an EA needs to cooperate with other EAs to achieve its goal. In addition, it could be trigged from the "joining a cooperation" state if a neighbor EA decides to cooperate or to answer positively a cooperation request. For example, when the TA of X waits for the cooperation of at least two other TAs.

An example of the data carrying method within the network and the cooperation message that summarizes the information of several SNs has been given in some of our previous works [6] [7]. 


\subsection{Agent Strategy}

Previously, we explained the importance of cooperating, but indeed, in some cases, not to join a cooperation could be better than joining it. For example, if the agent $\mathrm{B}$ does not have a lot of energy, $\mathrm{B}$ will prefer to cooperate for the fire detection instead of cooperating with X to localize the object. Hence, agent B gives more pritority to its MA than its TA. Therefore, each EA should computes the relevance $(\mathrm{R})$ of each cooperation. Thus, we defined four parameters: the energy E, the network density $\mathrm{D}$, the position of an $\mathrm{SN}$ within the network $\mathrm{P}$, and the information importance degree I, to compute the cooperation of each relevance. To simplify the computation of this relevance, we propose the equation (10).

$$
R=E \times \alpha+\frac{1}{D} \times \beta+P \times \theta+I \times \omega
$$

Where $\alpha, \beta, \theta$, and $\omega$ are the influence factors for the energy, the density, the position, and the information importance degree respectively. This strategy has been detailed in one of our previous work 8 .

\section{Results Discussion}

To the best of our knowledge, we do not see any work treating the data aggregation for a multi-application WSN. Therefore, to evaluate the relevance of our proposal (MADA), we suggest to evaluate its performance compared to a well known data aggregation approach, which is the data fusion [9. This approach could be described as follows. When a node sends its information to the sink, intermediate nodes merge their information with the first node information. As the information of multiple sensor nodes are merged into one message (one overhead instead of many ones), this solution reduces the information communications' power consumption.

We have implemented these approaches on Glomosim [10, which is a scalable simulation environment for wireless and wired network systems. The simulations parameters, which are related to the capacity of the SNs, have been defined according to the recommendation of sun SPOT 2] and [11]. The main simulations parameters are summarized in Table 1

\subsection{Power Consumption}

Fig 3 presents the average power consumption per node in the case of 1 and 3 applications in data fusion and MADA. The results point out that MADA decreases significantly the average power consumption of an $\mathrm{SN}$ in both scenarios ( 1 and 3 applications). In addition, we can observe that to deal with 3 applications instead of only one application in MADA, an SN consumes approximately an additional power equal to $2 \mathrm{~mJ}$. However, in data fusion, the aditional power is approximately equal to $27 \mathrm{~mJ}$.

These results prove also that for larger WSN, MADA maintains the SNs' consumption to low values, while the difference with data fusion becomes greater. 
Table 1. Simulation parameters

\begin{tabular}{|l|l|}
\hline Simulation Parameter & Values \\
\hline Network size & $5000 \mathrm{~m} \times 5000 \mathrm{~m}$ \\
Node distribution & Random \\
Radio range & $87 \mathrm{~m}$ \\
Throughput & $1 \mathrm{Mbps}$ \\
Size of sensed data & 24 byte per node \\
Sensed Data Interval & 10 seconds \\
Simulation time & 1000 seconds \\
$\alpha, \theta, \omega$ & 0.25 \\
$\beta$ & 0.1 \\
\hline
\end{tabular}

Hence, these results prove the suitability of MADA for small and large scale WSNs.

\subsection{End-to-End Delay}

This parameter represents the average delay needed to carry a message from a source node till the sink, including the time of communication and processing. The performance of MADA and data fusion corresponding to this criterion is presented in Fig. 4. The obtained results show that MADA requires more latency than data fusion. This extra latency is due to the required cooperation time in MADA. However, we could mention that the difference between MADA and data fusion is never more than $150 \mathrm{~ms}$. This difference means that only the application which requires more than 0.15 seconds will be influenced. We can point out also that the number of running applications does not influence the delay (delay for 1 or 3 applications is the same). Otherwise, in term of scalability, the strobe of the curves show that both approaches are scalable.

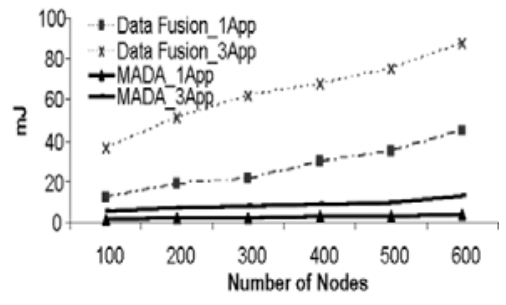

Fig. 3. Average power consumption per node

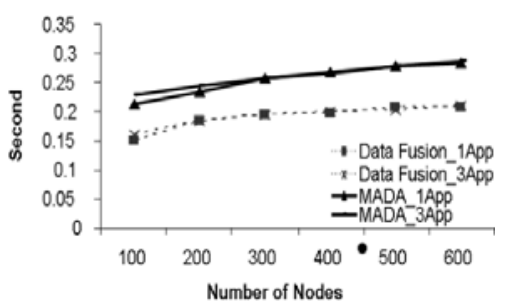

Fig. 4. Average end-to-end delay

\section{Conclusion}

In this paper, we have proposed a data aggregation scheme to optimize the power consumption of the sensor nodes, in the context of one and several applications deployed over the same WSN. This solution (MADA) is based on a multi-agent 
system to reduce the amount of communicated information by processing and filtering the information locally. MADA defines also a method based on several important parameters to decide the relevance of a cooperation. In addition, this method takes into consideration the priority of some applications compared to others ones (e.g. fire detection could be more important than object tracking if they are happening at the same time). MADA presents, through the results of the several simulations, a very good performance in term of power consumption.

Our future work will be based firstly on reducing the end-to-end latency. Then, we will optimize our agent strategy by integrating a more sophisticated mathematical model, which evaluates better the importance of each parameter.

\section{References}

1. Sangeeta, B., Chenyang, L., Gruia-Catalin, R.: Utility-based multi-application deployment in shared wireless sensor networks. Technical report, Washington University in St. Louis (2008)

2. Sun: Sun ${ }^{\text {TM }}$ Small Programmable Object Technology (Sun SPOT) Theory of Operation. Technical report, Sun Microsystem, Sun Labs (2008)

3. Levis, P., Gay, D., Culler, D.: Active sensor networks. In: NSDI, pp. 343-356. USENIX Association, Berkeley (2005)

4. Levis, P., Culler, D.: Maté: a tiny virtual machine for sensor networks. In: ASPLOSX, pp. 85-95. ACM, New York (2002)

5. Yu, Y., Rittle, L.J., Bhandari, V., LeBrun, J.B.: Supporting concurrent applications in wireless sensor networks. In: SenSys., pp. 139-152. ACM, New York (2006)

6. Sardouk, A., Merghem-Boulahia, L., Gaiti, D.: Agent-Cooperation Based Communication Architecture for Wireless Sensor Network. In: IFIP Wireless Days/Ad-hoc and Wireless Sensor Networks, UAE, IFIP (2008)

7. Sardouk, A., Rahim-Amoud, R., Merghem-Boulahia, L., Gaiti, D.: InformationImportance Based Communication for Large-Scale WSN Data Processing. In: WMNC, Gdansk,Poland, IFIP (2009)

8. Sardouk, A., Rahim-Amoud, R., Merghem-Boulahia, L., Gaiti, D.: A Strategy for Multi-Agent Based Wireless Sensor Network Optimization. In: Sadre, R., Pras, A. (eds.) AIMS 2009. IFIP International Federation for Information Processing 2009. LNCS, vol. 5637, pp. 122-133. Springer, Heidelberg (2009)

9. Das, S.R., Nasipuri, A., Patil, S.: Serial Data Fusion Using Space-filling Curves in Wireless Sensor Networks. In: IEEE, ed.: First Annual IEEE Communications Society Conference on Sensor and Ad Hoc Communications and Networks, pp. $182-190(2004)$

10. Laboratory, U., Laboratory, W.: Glomosim: A scalable simulation environment for wireless and wired network systems. In: The 3rd International Working Conference on Performance Modeling and Evaluation of Heterogeneous Networks (2005)

11. Sohraby, K., Minoli, D., Znati, T.: Wireless Sensor Networks, Technology, Protocols, and Applications. Willey, Chichester (2007) 